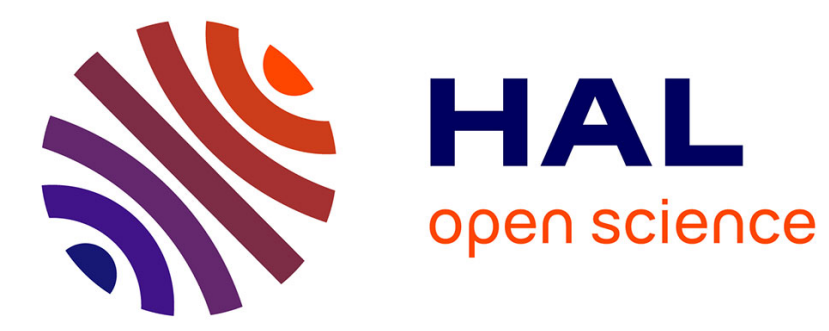

\title{
Continuation of discrete breathers from infinity in a nonlinear model for DNA breathing
}

\author{
Guillaume James, Antoine Levitt, Cynthia Ferreira
}

\section{To cite this version:}

Guillaume James, Antoine Levitt, Cynthia Ferreira. Continuation of discrete breathers from infinity in a nonlinear model for DNA breathing. Applicable Analysis, 2010, Mathematics of Nonlinear Lattices, 89 (9), pp.1447-1465. 10.1080/00036810903437788 . hal-00765599

\section{HAL Id: hal-00765599 \\ https://hal.science/hal-00765599}

Submitted on 2 Sep 2019

HAL is a multi-disciplinary open access archive for the deposit and dissemination of scientific research documents, whether they are published or not. The documents may come from teaching and research institutions in France or abroad, or from public or private research centers.
L'archive ouverte pluridisciplinaire HAL, est destinée au dépôt et à la diffusion de documents scientifiques de niveau recherche, publiés ou non, émanant des établissements d'enseignement et de recherche français ou étrangers, des laboratoires publics ou privés. 


\title{
Continuation of discrete breathers from infinity in a nonlinear model for DNA breathing
}

\author{
Guillaume James $^{\mathrm{a} *}$, Antoine Levitt ${ }^{\mathrm{a}}$ and Cynthia Ferreira ${ }^{\mathrm{b}}$ \\ ${ }^{a}$ Laboratoire Jean Kuntzmann, Université de Grenoble and CNRS, BP 53, 38041 Grenoble \\ Cedex 9, France; ${ }^{b}$ Département de Mathématiques, Institut de Mathématiques de \\ Toulouse, UMR 5219, INSA de Toulouse, 135 avenue de Rangueil, 31077 Toulouse \\ Cedex 4, France
}

\begin{abstract}
We study the existence of discrete breathers (time-periodic and spatially localized oscillations) in a chain of coupled nonlinear oscillators modelling the breathing of DNA. We consider a modification of the Peyrard-Bishop model introduced by Peyrard et al. [Nonlinear analysis of the dynamics of DNA breathing, J. Biol. Phys. 35 (2009), 73-89], in which the reclosing of base pairs is hindered by an energy barrier. Using a new kind of continuation from infinity, we prove for weak couplings the existence of large amplitude and low frequency breathers oscillating around a localized equilibrium, for breather frequencies lying outside resonance zones. These results are completed by numerical continuation. For resonant frequencies (with one multiple belonging to the phonon band) we numerically obtain discrete breathers superposed on a small oscillatory tail.
\end{abstract}

Keywords: discrete breathers; Peyrard-Bishop model for DNA; continuation from infinity; numerical continuation; resonances with phonons; breathers with oscillatory tails

AMS Subject Classifications: 37L60; 37K60; 92B99; 34C25; 34C37

\section{Introduction}

The base pairs of DNA show large amplitude localized fluctuations known as the breathing of DNA. Despite the complexity of real DNA, this phenomenon is reproduced by a much simpler nonlinear model discovered by Peyrard and Bishop $[1,2]$. This model considers only one variable for each base pair, the stretching $y_{n}$ of hydrogen bonds connecting the two bases with index $n$ (Figure 1). It is defined by the Hamiltonian

$$
H=\sum_{n \in \mathbb{Z}} \frac{p_{n}^{2}}{2 m}+W\left(y_{n}, y_{n-1}\right)+V\left(y_{n}\right), \quad \text { with } p_{n}=m \frac{\mathrm{d} y_{n}}{\mathrm{~d} t},
$$

*Corresponding author. Email: guillaume.james@imag.fr 

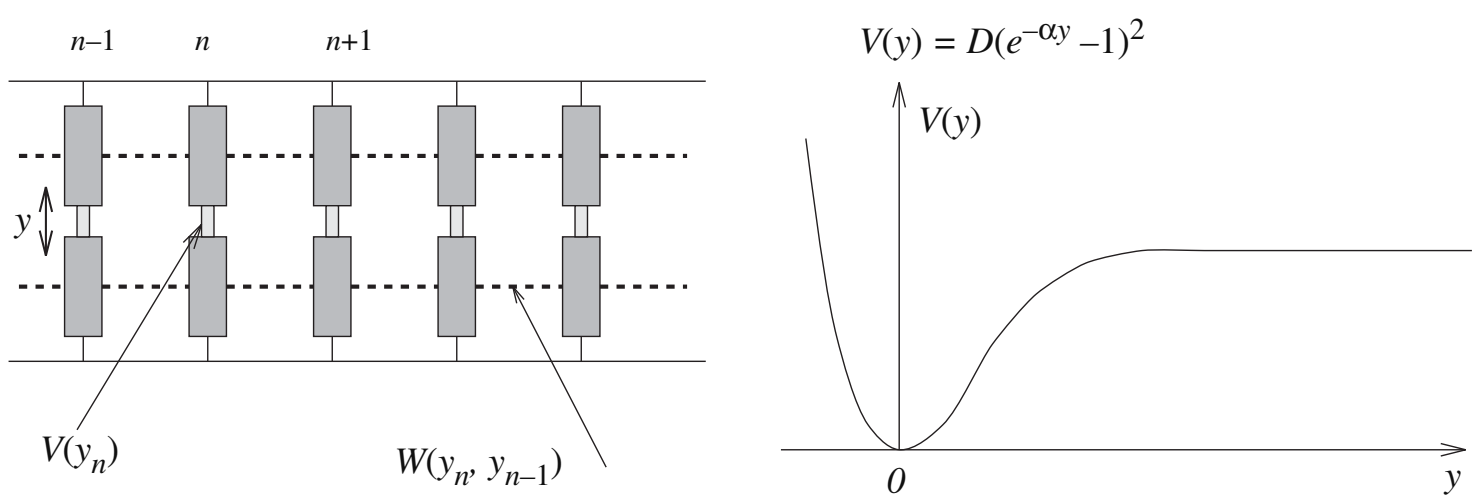

Figure 1. Peyrard-Bishop model for DNA nonlinear dynamics, described by Hamiltonian (1).

for an homogeneous DNA molecule. The interaction between two bases in a pair is described by the potential $V$. The Peyrard-Bishop model considers a Morse potential represented in Figure 1 (the plateau of $V$ corresponds to the dissociation of a base pair for large $y_{n}$ ). The potential $W$ accounts for interactions between adjacent bases. In what follows we consider a harmonic potential $W\left(y_{n}, y_{n-1}\right)=\frac{\gamma}{2}\left(y_{n}-y_{n-1}\right)^{2}$ $(\gamma>0)$ for simplicity, but nonlinear coupling effects should be introduced to describe correctly the thermal denaturation of DNA [3].

The Peyrard-Bishop model exhibits spatially localized oscillations denoted as discrete breathers [2], which qualitatively correspond to DNA breathing. Such excitations are common in spatially discrete and nonlinear systems [4], where nonlinearity can lead to energy localization via modulational instability and inelastic collisions of wave packets [5,6]. Discrete breathers studied in experiments or numerical simulations consist of a large class of spatially localized oscillations, such as metastable states, oscillations with a certain degree of periodicity or even chaotic oscillations interacting with an extended background. Conversely, mathematical results on their existence mostly concern time-periodic oscillations (see [7-15] and references therein).

From a physical point of view, the link between DNA breathing and discrete breathers is not yet firmly established because the fluctuations detected in experiments and in simulations of the Peyrard-Bishop model are different by several orders of magnitude [16,17]. However, progress has been made in this direction since a recent improvement of the model was able to bring some key dynamical properties in the range measured in experiments [17]. In the new model, the Morse potential is replaced by a more elaborate one including an energy barrier for reclosing (Figure 2). The new potential satisfies $V^{\prime}(0)=0, V^{\prime \prime}(0)>0$ and possesses a unique local maximum at $y_{b} \in(0,1)$. We have in addition

$$
V(y)=D+E e^{-\beta y}\left(y+\frac{1}{\beta}\right) \text { if } y>1,
$$

where $y$ is expressed in $\AA$ in the physical model [16,17]. The barrier for reclosing has several physical origins. Firstly, all-atom molecular dynamics simulations show that open bases can fluctuate a lot, their motions including rotations which may hinder the reclosing of the pairs. In model (1) where only a subset of the degrees of freedom of the system are described, this effect can be included via an energy cost for closing. Indeed, the potentials of (1) are actually free energies that take into account all the degrees of freedom which are not included in the model. Reclosing of a base pair will 


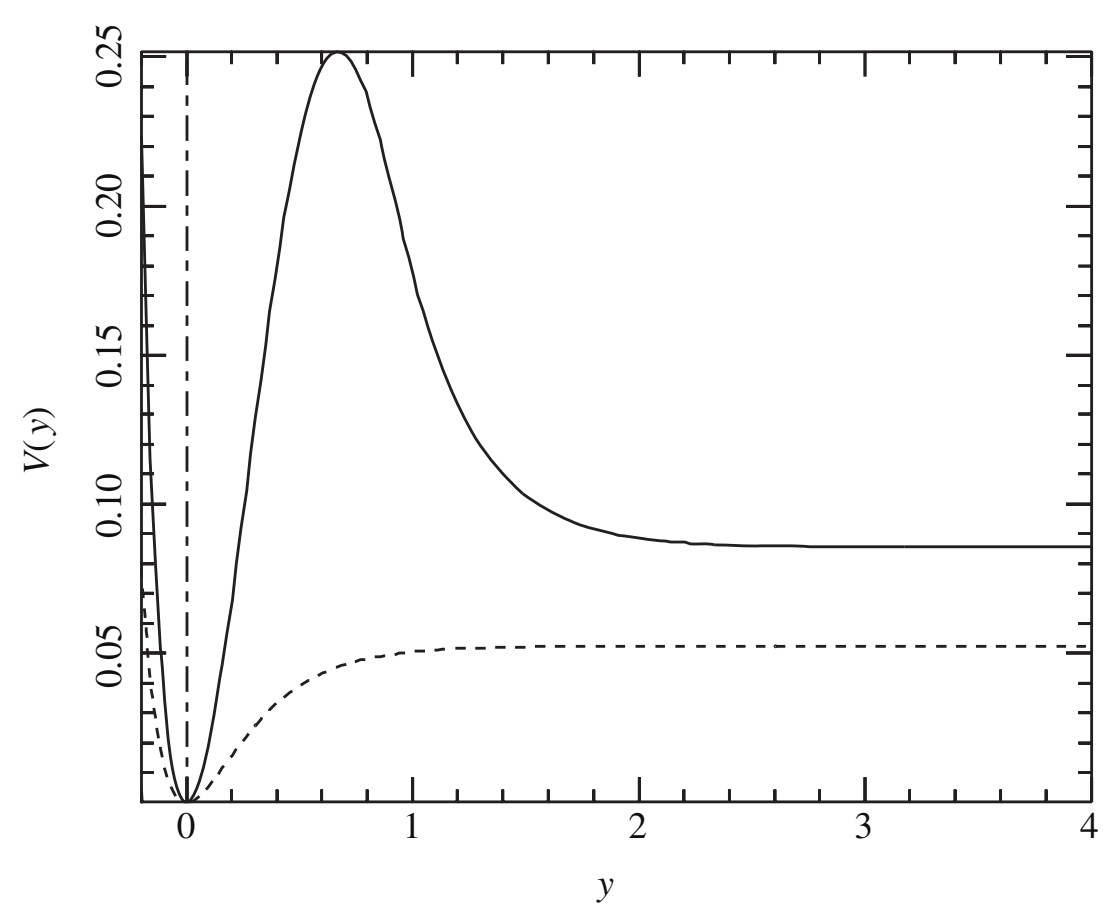

Figure 2. Potential $V$ with barrier for reclosing (full line), compared with a Morse potential (dashed line).

decrease the entropy, bringing a gain in free energy. Secondly, open bases tend to form hydrogen bonds with water molecules in the solvent. These bonds have to be broken for closing, which gives an additional contribution to the energy barrier.

The model with potential barrier admits a new type of discrete breathers having a single excited site which oscillates beyond the barrier for all times. Such solutions were found numerically in reference [17]. In quantitative agreement with experimental results, they significantly increase the average lifetime of opening events, have a much stronger spatial localization and decrease the proportion of open base pairs by collecting more vibrational energy.

In this article, we prove the existence of spatially localized solutions which oscillate beyond the potential barrier and are time-periodic (see Theorem 2.2). This result is valid for small values of the coupling constant $\gamma$, and under a nonresonance condition stipulating that all multiples of the breather frequency $\omega_{b}$ lie outside the phonon band. As a consequence, breathers exist for parameters $\left(\omega_{b}, \gamma\right)$ lying outside certain resonance tongues accumulating near $(0,0)$.

Our proof is based on an extension of the concept of anticontinuum limit [12,14]. For $\gamma \approx 0$, breather solutions are proved to exist near a simple time-periodic localized excitation involving only one oscillator. Our analysis differs from the usual anticontinuum limit in several ways.

Firstly, when $\gamma=0$ the excited site lies at infinity on the plateau of the potential $V$. In other words, breathers are obtained by 'continuation from infinity' for arbitrarily small values of $\gamma$, but without reaching $\gamma=0$. Our perturbative analysis is performed near an approximate solution for $\gamma \approx 0$, instead of an exact solution for $\gamma=0$ as in the classical anticontinuum limit. Note that our result differs from previous works on continuation of periodic orbits from infinity, which concern finite-dimensional and asymptotically linear systems (see [18] and references therein).

Secondly, the maximal frequency of discrete breathers beyond the potential barrier goes to 0 with $\gamma$ and thus it is not possible to perform the continuation at 
fixed frequency as in [12]. Indeed, close to their maximal frequency, breathers beyond the barrier correspond to (weakly nonlinear) localized modes of a spatially localized equilibrium $y_{n}^{\mathrm{eq}}$. When $\gamma$ is small, $y_{0}^{\mathrm{eq}}$ goes to infinity on the flat part of the potential, which induces low-frequency oscillations.

Our analytical results are completed by numerical computations of discrete breathers, for parameter values corresponding to an homogeneous DNA molecule. We use the numerical method developed in $[19,20]$, i.e. breathers are defined as zeros of the time- $T$ map of the flow and computed using the Gauss-Newton method and path-following. In addition to the discrete breathers oscillating beyond the barrier (Theorem 2.2) and the classical ones oscillating below [11,12], we find a new family of breather solutions oscillating on both sides of the barrier. These solutions also appear from infinity as $\gamma \rightarrow 0$ and the proof of their existence is still an open problem (it does not follow from the present analysis which relies on specific scaling properties of breathers oscillating beyond the barrier). In addition, when a multiple of the breather frequency lies in the phonon band, we find that breathers are replaced by 'almost localized' solutions having a small oscillatory tail at infinity. Such solutions are known as phonobreathers or nanopterons (see [21] and references therein). They consist of a particular class of multibreathers [9], hence their existence at small coupling follows from the usual anticontinuum limit for oscillations below the potential barrier. However, along the new solution branches investigated here, the proof of their existence by continuation from infinity remains an open problem.

The outline of the article is as follows. In Section 2, we explain the idea of continuation from infinity at low coupling for localized equilibria and discrete breathers and state our main results. The existence of discrete breathers that oscillate beyond the barrier is proved in Section 3, and numerical computations are performed in Section 4.

\section{Anti continuum limit with small restoring force}

We assume that the on-site potential $V$ belongs to $C^{k+1}(\mathbb{R})$ with $k$ large enough. After a rescaling, the dynamical equations of (1) are recast in the form

$$
\frac{\mathrm{d}^{2} y_{n}}{\mathrm{~d} t^{2}}+V^{\prime}\left(y_{n}\right)=\gamma\left(y_{n+1}-2 y_{n}+y_{n-1}\right), \quad n \in \mathbb{Z},
$$

where $V^{\prime}(0)=0, V^{\prime \prime}(0)=1$.

We begin by introducing in Section 2.1 the idea of continuation from infinity at low coupling, in the simplest case of equilibria (following reference [17]). The case of discrete breathers is more delicate due to the existence of resonances, and will be examined in Sections 2.2 (construction of approximate breather solutions) and 2.3 (existence theorem).

\subsection{Continuation of a localized equilibrium from infinity}

A spatially localized equilibrium of (3) was obtained in [17] by continuation from infinity, for small enough values of $\gamma$. It corresponds to a solution of

$$
\gamma\left(y_{n+1}-2 y_{n}+y_{n-1}\right)=V^{\prime}\left(y_{n}\right), \quad n \in \mathbb{Z}
$$


satisfying

$$
\lim _{n \rightarrow \pm \infty} y_{n}=0 .
$$

The equilibrium is spatially symmetric, with $y_{-n}=y_{n}$ for all $n \in \mathbb{Z}$. It satisfies in addition

$$
\lim _{\gamma \rightarrow 0} y_{0}=+\infty, \quad \lim _{\gamma \rightarrow 0} y_{n}=0 \quad \text { for } n \neq 0 .
$$

This solution is constructed using an extension of the technique of anticontinuum limit for maps $[22,23]$. In the classical anticontinuum limit one starts from an exact solution of (4)-(5) for $\gamma=0$ ( $y_{n}$ is then a critical point of $V$ for any $\left.n\right)$ and one uses the implicit function theorem to continue this solution to $\gamma \approx 0$. In the limit case $\gamma=0$ of (6), the critical point $y_{0}$ lies at infinity and the problem becomes singular. However, a solution of (4)-(6) can be found in the vicinity of a suitable approximate solution $\tilde{y}_{n}$. This initial guess satisfies $\tilde{y}_{n}=0$ for $n \neq 0$, and $\tilde{y}_{0}$ is a critical point of the modified potential

$$
V_{\gamma}(y)=V(y)+\gamma y^{2},
$$

which incorporates a restoring force due to nearest-neighbours (Figure 3).

With the particular potential (2) and for $\gamma$ small enough, the solution of $V_{\gamma}^{\prime}\left(\tilde{y}_{0}\right)=0$ in the interval $[1, \infty)$ reads explicitly

$$
\tilde{y}_{0}=-\frac{1}{\beta} \ln \left(\frac{2 \gamma}{\beta E}\right) \text {. }
$$

An exact solution $y_{n}^{\text {eq }}$ of (4)-(5) satisfying (6) is obtained in [17] for $\gamma \approx 0$, using the contraction mapping theorem in some neighbourhood of $\tilde{y}_{n}$. This solution has the following asymptotic form ( $\delta_{m, n}$ denotes the usual Kronecker symbol).

THEOREM 2.1 [24] There exists a constant $\gamma_{0}>0$ such that for all $\gamma \in\left(0, \gamma_{0}\right)$, Problem (4)-(5) admits a solution $y_{n}^{\mathrm{eq}}(\gamma)$ satisfying

$$
\operatorname{Sup}_{n \in \mathbb{Z}}\left|y_{n}^{\mathrm{eq}}(\gamma)-\tilde{y}_{n}(\gamma)\right|=O(|\gamma \ln (\gamma)|), \quad \gamma \rightarrow 0,
$$

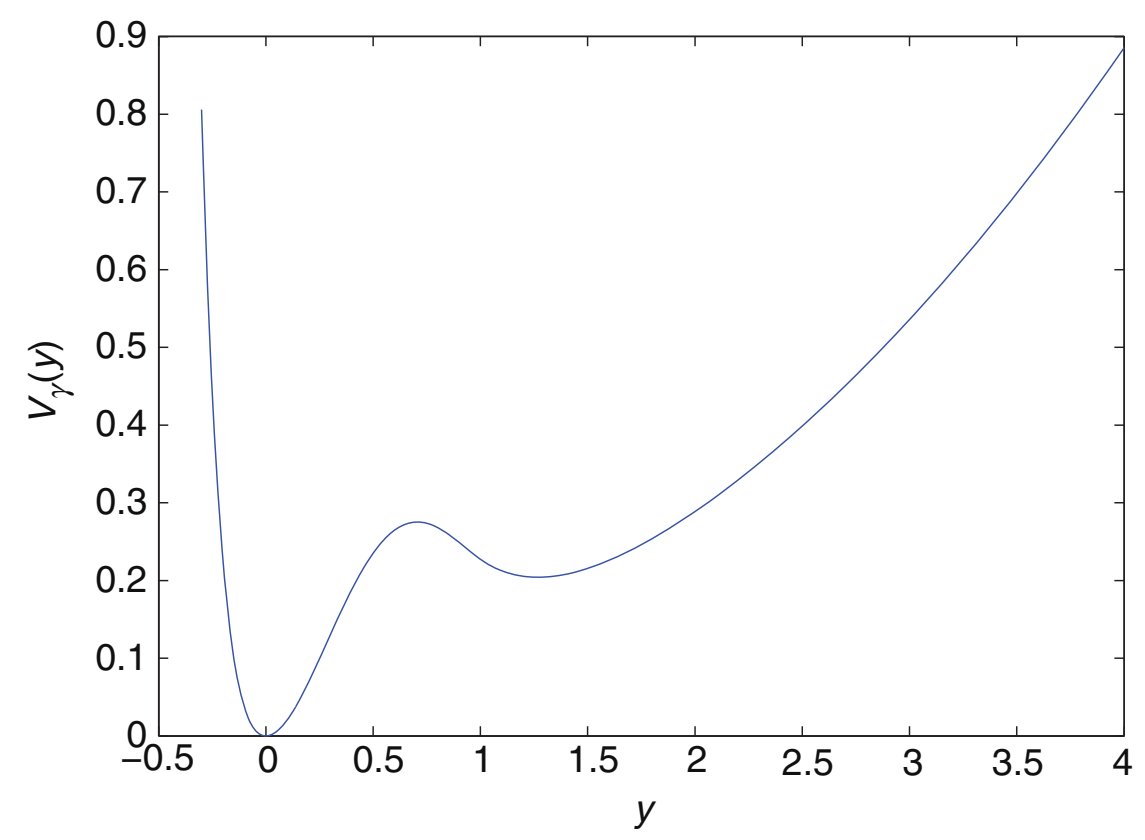

Figure 3. Potential $V_{\gamma}(y)=V(y)+\gamma y^{2}$. 
where $\tilde{y}_{n}(\gamma)=-\frac{1}{\beta} \ln \left(\frac{2 \gamma}{\beta E}\right) \delta_{n, 0}$. Moreover, $y_{n}^{\mathrm{eq}}$ decays to 0 exponentially as $n \rightarrow \pm \infty$ and has the symmetry $y_{-n}^{\mathrm{eq}}=y_{n}^{\mathrm{eq}}$.

With the same kind of technique, we shall prove for $\gamma \approx 0$ the existence of breathers oscillating beyond the potential barrier at $y_{b}$, in some finite-size neighbourhood of $\tilde{y}_{n}$.

\subsection{Approximate breather solutions and resonances}

We first establish for $\gamma \approx 0$ the asymptotic form of breather oscillations in a finitesize neighbourhood of $\tilde{y}_{n}$. For $n=0$ one rewrites Equation (3) as

$$
\frac{\mathrm{d}^{2} y_{0}}{\mathrm{~d} t^{2}}+V_{\gamma}^{\prime}\left(y_{0}\right)=\gamma\left(y_{1}+y_{-1}\right)
$$

In order to approximate a highly localized breather centred at $n=0$, we set $y_{1}=y_{-1}=0$ and obtain

$$
\frac{\mathrm{d}^{2} y_{0}}{\mathrm{~d} t^{2}}+V_{\gamma}^{\prime}\left(y_{0}\right)=0
$$

Let us examine problem (10) in a neighbourhood of the equilibrium $\tilde{y}_{0}$ given by (7). Assuming $y_{0}(t)>1$ for all $t \in \mathbb{R}$ and setting $y_{0}(t)=\tilde{y}_{0}+\tilde{x}(t)$, we get

$$
V_{\gamma}\left(\tilde{y}_{0}+\tilde{x}\right)=\tilde{D}(\gamma)-2 \gamma \ln (\gamma) \Phi(\tilde{x})+2 \gamma U(\tilde{x})
$$

where $\tilde{D}(\gamma)$ is independent of $\tilde{x}, \Phi(x)=\frac{1}{\beta^{2}}\left(e^{-\beta x}-1+\beta x\right)$ and $U$ is an analytic function satisfying $U(0)=U^{\prime}(0)=0$. For solutions of (10), the frequency of oscillations around $\tilde{y}_{0}$ in the limit of vanishing amplitude is therefore $\sqrt{V_{\gamma}^{\prime \prime}\left(\tilde{y}_{0}\right)}=\sqrt{-2 \gamma \ln (\gamma)}\left(1+O\left(|\ln \gamma|^{-1}\right)\right)$. Neglecting $O(\gamma)$ terms in (11) and setting $\tilde{x}(t)=x(\sqrt{-2 \gamma \ln (\gamma)} t)$, problem (10) yields the renormalized equation

$$
\frac{\mathrm{d}^{2} x}{\mathrm{~d} t^{2}}+\Phi^{\prime}(x)=0
$$

where $\Phi^{\prime}(0)=0, \Phi^{\prime \prime}(0)=1$.

The potential $\Phi$ is soft, i.e. the frequency $\omega(A)$ of periodic solutions $x$ of (12) is a decreasing function of their amplitude $A=\|x\|_{\infty}$ (this property will be important for our continuation procedure, see Theorem 2.2). At small amplitudes this result follows from the classical formula [25, Section 26, p. 241]

$$
\omega=1+\frac{1}{16} h A^{2}+o\left(A^{2}\right), \quad h=\Phi^{(4)}(0)-\frac{5}{3}\left(\Phi^{(3)}(0)\right)^{2}=-\frac{2}{3} \beta^{2}<0 .
$$

At large amplitudes, we use the fact that the period $T=2 \pi / \omega$ of a solution $x$ with energy $H=\frac{\dot{x}^{2}}{2}+\Phi(x)$ satisfies

$$
T=\sqrt{2} \int_{a(H)}^{b(H)} \frac{\mathrm{d} x}{\sqrt{H-\Phi(x)}},
$$

where $a(H)<0$ and $b(H)>0$ are implicitly defined by $\Phi(a)=\Phi(b)=H$. It follows that $T \sim 2 \sqrt{2} \sqrt{H}$ as $H \rightarrow+\infty$ (split (13) into two integrals for $x<0$ and $x>0$ and use the 
change of variables $\Phi(x)=y$ in each part), hence $T$ increases with energy (or equivalently amplitude) at large amplitudes. In addition, we have checked numerically that this property remains true at finite amplitudes.

As a conclusion, an approximate breather solution of (3) for $\gamma \approx 0$ is given by

$$
y_{n}^{*}(t)=\delta_{n, 0}\left[-\frac{1}{\beta} \ln \left(\frac{2 \gamma}{\beta E}\right)+x(\sqrt{-2 \gamma \ln (\gamma)} t)\right],
$$

where $x$ is time-periodic (with frequency $\omega<1$ ) and satisfies (12). When $\gamma \rightarrow 0$, the amplitude of this approximate solution goes to infinity and its frequency goes to 0 .

Now this raises the question of existence of an exact breather solution of (3) in the vicinity of $y_{n}^{*}$. An important condition for the existence of breathers is nonresonance with linear waves [12,21]. Equation (3) linearized at $y_{n}=0$ admits solutions in the form of linear waves (phonons) $y_{n}(t)=A \cos \left(q n-\omega_{q} t\right)$, whose frequency satisfies the dispersion relation

$$
\omega_{q}^{2}=1+2 \gamma(1-\cos q) .
$$

Phonon frequencies belong to the band $[1, \sqrt{1+4 \gamma}]$, and the nonresonance condition requires that all multiples of the breather frequency lie outside this interval. Figure 4 depicts the nonresonant breather frequencies $\omega_{b}$ when $\gamma$ is varied (shaded regions). Resonance tongues accumulate near $\left(\omega_{b}, \gamma\right)=(0,0)$, and the $m$-th and $(m+1)$-th tongues merge at a vertex $V_{m}=\left(m^{-1}, g_{m}\right)$ with $g_{m}=(2 m)^{-1}+(2 m)^{-2}$.

In order to obtain an exact breather solution, we must firstly determine when the approximate solution $y_{n}^{*}$ is itself nonresonant. Let us fix a solution $x$ of (12) with frequency $\omega<1$. The corresponding approximate breather solution (14) has frequency $\omega^{*}(\gamma)=\omega \sqrt{-2 \gamma \ln (\gamma)}$ (maximal for $\gamma=e^{-1}$ ), which defines a graph in

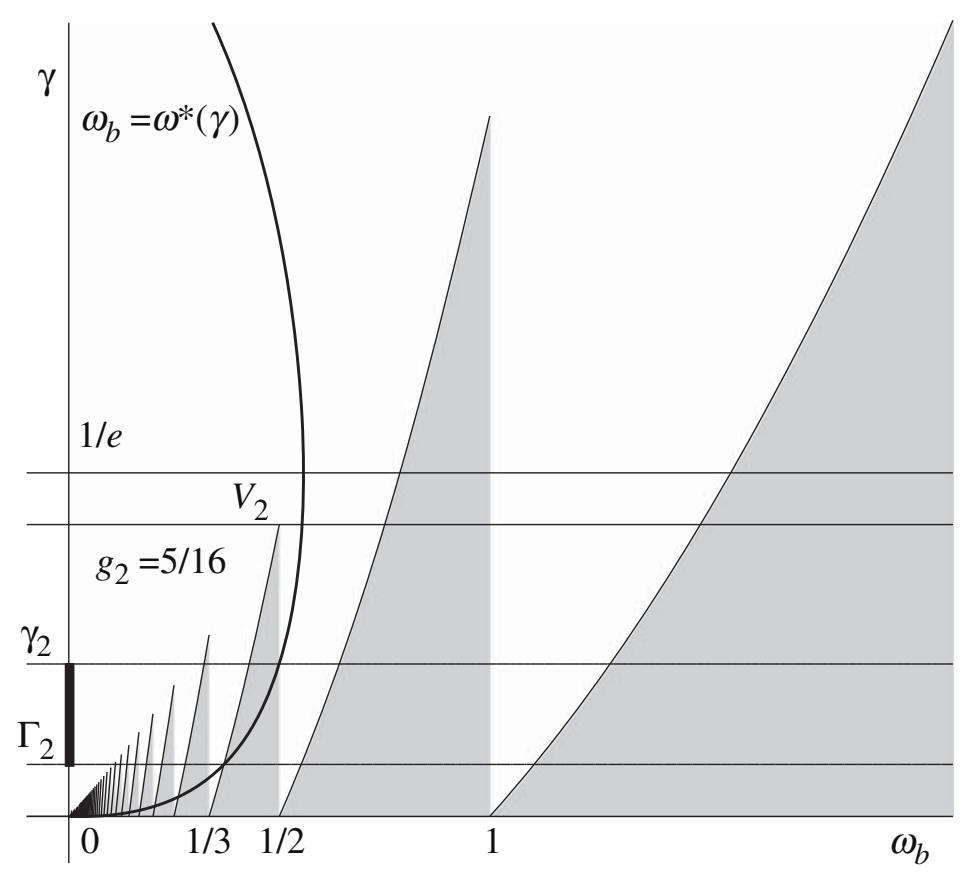

Figure 4. Nonresonant breather frequencies $\omega_{b}$ for different values of $\gamma$ (shaded regions). Resonance tongues (white regions) correspond to the phonon band and its submultiples. The curve provides the frequency $\omega_{b}=\omega^{*}(\gamma)$ of the approximate breather solution (14), for different values of $\gamma$ and the particular value $\omega=0.65$. 
the parameter plane (Figure 4). Let us denote by $m_{0}(\omega) \geq 2$ the smallest integer $m$ such that $\omega^{*}\left(g_{m}\right)>m^{-1}$. For all integers $m \geq m_{0}$, critical coupling values $\gamma_{m} \leq e^{-1}$ and $\Gamma_{m} \leq \gamma_{m}$ are implicitly defined by the relations

$$
\omega^{*}\left(\gamma_{m}\right)=\frac{1}{m}, \quad \omega^{*}\left(\Gamma_{m}\right)=\frac{\sqrt{1+4 \Gamma_{m}}}{m+1},
$$

and the approximate breather solution (14) is nonresonant for $\gamma \in\left(\Gamma_{m}, \gamma_{m}\right)$ (Figure 4). This yields an infinity of disjoint intervals of nonresonance accumulating at $\gamma=0$, since $\lim _{m \rightarrow+\infty} \gamma_{m}=0$ and $\lim _{m \rightarrow+\infty} \Gamma_{m}=0$.

In the next section, we state the main result of this article, i.e. the existence of exact breather solutions close to the family of approximate solutions (14) parametrized by $\gamma \approx 0$ ( $x$ or equivalently $\omega$ being fixed). As previously explained, breather continuation cannot be performed in a connected neighbourhood of $\gamma=0$ because of resonances. We shall prove instead the existence of breathers in an infinity of disjoint intervals $\left[\tilde{\Gamma}_{m}, \tilde{\gamma}_{m}\right] \subset\left(\Gamma_{m}, \gamma_{m}\right)$ accumulating at $\gamma=0$. We do not have an existence result in the entire nonresonance intervals $\left(\Gamma_{m}, \gamma_{m}\right)$ because our proof is based on the contraction mapping theorem, which cannot be applied too close to resonances.

\subsection{Main results}

In this section we assume $V$ of class $C^{k+1}$ with $k \geq 4$. We shall prove the existence of exact breather solutions of (3) close to the ansatz (14), for small enough values of $\gamma$ in a subset of $\mathcal{C}_{\omega}=\cup_{m \geq m_{0}(\omega)}\left(\Gamma_{m}, \gamma_{m}\right)$ defined as follows (we introduce an appropriate cut-off of nonresonant frequency intervals, and deduce a suitable cut-off of the nonresonant coupling intervals $\left.\left(\Gamma_{m}, \gamma_{m}\right)\right)$.

For $\gamma=0$, the bands of nonresonant frequencies correspond to the intervals $(1,+\infty)$ and $\left((m+1)^{-1}, m^{-1}\right)$ for all integers $m \geq 1$ (Figure 4). We introduce

$$
\Omega_{m}=\left[m^{2} \alpha(m)+(m+1)^{2}(1-\alpha(m))\right]^{-1 / 2}, \quad \omega_{m}=\left[m^{2}(1-\alpha(m))+(m+1)^{2} \alpha(m)\right]^{-1 / 2},
$$

where the function $\alpha$ is defined by

$$
\alpha(s)= \begin{cases}\alpha_{0} & \text { for } 0 \leq s \leq e, \\ \alpha_{0}(\ln s)^{-r} & \text { for } s \geq e\end{cases}
$$

with $\alpha_{0}, r \in\left(0, \frac{1}{2}\right)$. Clearly the frequencies $\Omega_{m}, \omega_{m}$ lie between the resonant frequencies $(m+1)^{-1}, m^{-1}$, with $\Omega_{m}<\omega_{m}$ (since $\left.\alpha<1 / 2\right)$. Moreover, the decay of $\alpha(m)$ implies $m^{-1}-\omega_{m}=o\left(m^{-2}\right)$ as $m \rightarrow+\infty$, i.e. the distance between $\omega_{m}$ and $m^{-1}$ is small compared to the band of nonresonant frequencies $\left((m+1)^{-1}, m^{-1}\right)$ (the same holds true for $\Omega_{m}$ and $\left.(m+1)^{-1}\right)$. The power-logarithmic decay of $\alpha$ introduced in (18) originates from the relative sizes of the first and second terms of (11) and the fixedpoint technique employed in the existence proof (Section 3). The whole proof could be carried out for $r=0$ in (18) (i.e. for constant $\alpha$ ), but choosing $r>0$ yields an existence result for a larger set of parameters.

Now we consider the solutions $\tilde{\gamma}_{m}, \tilde{\Gamma}_{m}$ of

$$
\omega^{*}\left(\tilde{\gamma}_{m}\right)=\omega_{m}, \quad \omega^{*}\left(\tilde{\Gamma}_{m}\right)=\Omega_{m} .
$$


When $m \rightarrow+\infty$, it is lengthy but straightforward to check that

$$
\Omega_{m}=\omega^{*}\left(\Gamma_{m}\right)\left[1+\alpha_{0} m^{-1}(\ln m)^{-r}+o\left(m^{-1}(\ln m)^{-r}\right)\right]>\omega^{*}\left(\Gamma_{m}\right)
$$

(use the identity $m+1=\sqrt{1+4 \Gamma_{m}}\left(\omega^{*}\left(\Gamma_{m}\right)\right)^{-1}$ in the definition of $\Omega_{m}$ ). Consequently we have for $m$ large enough $\left[\Omega_{m}, \omega_{m}\right] \subset\left(\omega^{*}\left(\Gamma_{m}\right), \omega^{*}\left(\gamma_{m}\right)\right.$ ) (band of nonresonant breather frequencies along the graph of $\left.\omega^{*}\right)$, which implies $\left[\tilde{\Gamma}_{m}, \tilde{\gamma}_{m}\right] \subset\left(\Gamma_{m}, \gamma_{m}\right)$. As a conclusion, for $m$ large enough and $\gamma \in\left[\tilde{\Gamma}_{m}, \tilde{\gamma}_{m}\right]$, the approximate breather solution (14) is nonresonant i.e. no multiple of its frequency belongs to the phonon band. Moreover, for $\gamma \in\left[\tilde{\Gamma}_{m}, \tilde{\gamma}_{m}\right]$ one stays far enough from resonances to obtain the following existence result (Section 3).

In what follows we note $H_{p e r}^{p}(0, T)$ the classical Sobolev space of $T$-periodic functions with distributional derivatives up to order $p$ belonging to $L_{p e r}^{2}(0, T)$. We also consider $H_{e}^{p}(0, T)=\left\{y \in H_{p e r}^{p}(0, T), y\right.$ is even $\}$ and define $L_{e}^{2}(0, T)$ similarly.

THeOREM 2.2 Fix a solution $x \in H_{e}^{2}(0, T)$ of (12), with period $T>2 \pi$ given by (13) and satisfying $T^{\prime}(H)>0$. Let $y_{n}^{*}$ denote the approximate breather solution of (3) defined by (14) for $\gamma \approx 0$, with frequency $\omega^{*}(\gamma)=2 \pi \sqrt{-2 \gamma \ln (\gamma)} / T$. Let $\tilde{\Gamma}_{m}, \tilde{\gamma}_{m}$ denote the coupling values defined by (17)-(19) (for a fixed $r \in\left(0, \frac{1}{2}\right)$ ) and satisfying $\lim _{m \rightarrow+\infty} \tilde{\gamma}_{m}=0, \lim _{m \rightarrow+\infty} \tilde{\Gamma}_{m}=0$. Then for a large enough integer $p$ and all coupling constants $\gamma$ in the set

$$
\mathcal{S}_{p}=\cup_{m \geq p}\left[\tilde{\Gamma}_{m}, \tilde{\gamma}_{m}\right]
$$

Equation (3) admits an exact breather solution $y_{n}$ close to (14). The solution $y_{n}$ has frequency $\omega^{*}(\gamma)$ and reads

$$
y_{n}(t)=y_{n}^{*}(t)+\xi_{n}(\sqrt{-2 \gamma \ln (\gamma)} t),
$$

with $\xi_{n} \in H_{e}^{2}(0, T)$ and $\xi_{-n}=\xi_{n}$ for all $n \in \mathbb{Z}$. The map $\gamma \mapsto\left(\xi_{n}\right)_{n \in \mathbb{Z}}$ is $C^{k}$ from $\mathcal{S}_{p}$ to $\ell_{\infty}\left(\mathbb{Z}, H_{e}^{2}(0, T)\right)$. One has in addition $\left\|\xi_{0}\right\|_{H^{2}}=O\left(|\ln \gamma|^{-1}\right)$ and there exist constants $C$, $K>0$ such that for all $n \neq 0$

$$
\left\|\xi_{n}\right\|_{H^{2}} \leq C\left(K|\ln \gamma|^{r-1 / 2} \gamma^{1 / 2}\right)^{|n|} .
$$

Note that Theorem 2.2 gives an existence result at small coupling since the integer $p$ in (21) must be chosen large enough. The breather solutions (22) decay exponentially in space according to estimate (23). They correspond to anharmonic oscillations (Equation (12)) in some finite-size neighbourhood of the localized equilibrium $y_{n}^{\mathrm{eq}}$. Consequently, our result goes beyond the weakly nonlinear regime accessible via the Lyapunov centre theorem.

The next section is devoted to the proof of Theorem 2.2.

\section{Proof of Theorem 2.2}

In order to prove Theorem 2.2, the first step is to reformulate (3) as a fixed point problem for the sequence $\left(\xi_{n}\right)_{n \in \mathbb{Z}}$ of time-periodic functions. We recall that $\xi_{n}$ is the small perturbation that separates the approximate breather solution (14) from the exact solution (22). Since $\xi_{-n}=\xi_{n}$ we shall only consider the semi-infinite sequence $\left(\xi_{n}\right)_{n \geq 0}$. Our analysis will be valid for small enough values of $\gamma$ and 
nonresonant breather frequencies $\omega^{*}(\gamma)$. This leads us to consider $\gamma$ in the set $\mathcal{S}_{p}$ defined by (21), where the integer $p$ is chosen large enough.

We first introduce a suitable scaling of $\xi_{n}$. To obtain the approximate solution (14) we have neglected the third term of potential (11), which scales as the second (principal) term times $|\ln \gamma|^{-1}$. Consequently we set $\xi_{0}=-(\ln \gamma)^{-1} u_{0}$ with $u_{0}=O(1)$ as $\gamma \rightarrow 0$. Moreover, substituting (22) in Equation (3) yields for $n=1$

$$
-2 \gamma \ln \gamma \frac{\mathrm{d}^{2} \xi_{1}}{\mathrm{~d} t^{2}}+\xi_{1}=\gamma \tilde{y}_{0}+\gamma\left(\xi_{2}-2 \xi_{1}\right)-\gamma(\ln \gamma)^{-1} u_{0}+O\left(\xi_{1}^{2}\right)+\gamma x .
$$

In order to equilibrate the two last terms of (24) we thus set $\xi_{1}=\sqrt{\gamma} u_{1}$. For simplicity we use the same scaling for all $n \geq 1$, setting $\xi_{n}=\sqrt{\gamma} u_{n}$. We search the sequence $u=\left(u_{n}\right)_{n \geq 0}$ in

$$
\mathcal{E}=\left\{u=\left(u_{n}\right)_{n \geq 0} \in \ell_{\infty}\left(\mathbb{N}_{0}, H_{e}^{2}(0, T)\right),\left\|u_{0}\right\|_{H^{2}} \leq \rho_{0},\left\|u_{n}\right\|_{H^{2}} \leq 1 \text { for all } n \geq 1\right\},
$$

where $\rho_{0}$ will be conveniently chosen. Since $\mathcal{E}$ is a closed subspace of $\ell_{\infty}\left(\mathbb{N}_{0}, H_{e}^{2}(0, T)\right)$, it defines a complete metric space for the distance associated with $\|u\|_{\infty}=\sup _{n \geq 0}\left\|u_{n}\right\|_{H^{2}}$.

In what follows we express $u$ as a fixed point of a suitable map $\mathcal{F}_{\gamma}$ defining a contraction on $\mathcal{E}$. Firstly, if $x, \rho_{0}$ are fixed and $\gamma$ is small enough then for all $u \in \mathcal{E}$ the sequence $\left(y_{n}\right)$ defined by (22) satisfies $y_{0}(t)>1$ for all $t \in \mathbb{R}$, and $y_{0}(t)$ lies beyond the potential barrier. Using Equation (9), expansion (11) and the spatial symmetry of $y_{n}$, we obtain

$$
\frac{\mathrm{d}^{2} u_{0}}{\mathrm{~d} t^{2}}+\Phi^{\prime \prime}(x) u_{0}=\epsilon^{-1} g_{0}\left(x, \epsilon u_{0}\right)-U^{\prime}\left(x+\epsilon u_{0}\right)+\sqrt{\gamma} u_{1},
$$

where $\epsilon(\gamma)=-(\ln \gamma)^{-1}$ (we shall often omit the argument $\gamma$ in notations) and $g_{0}\left(x, \xi_{0}\right)=-\Phi^{\prime}\left(x+\xi_{0}\right)+\Phi^{\prime}(x)+\Phi^{\prime \prime}(x) \xi_{0}=O\left(\xi_{0}^{2}\right)$ when $\xi_{0} \rightarrow 0$.

Now we consider the continuous linear operator $\mathcal{A}: H_{p e r}^{2}(0, T) \rightarrow L_{p e r}^{2}(0, T)$, $u \mapsto \frac{\mathrm{d}^{2} u}{\mathrm{~d} t^{2}}+\Phi^{\prime \prime}(x) u$ on the left-hand side of (25). Since $T^{\prime}(H) \neq 0$ on the periodic solution $x$ of (12) (we have assumed $T^{\prime}(H)>0$ in Theorem 2.2), it is a classical result that $\operatorname{Ker} \mathcal{A}$ is spanned by $x^{\prime}$ (see e.g. [14, Section 2.5]). Denoting by $\mathcal{A}_{e}$ the restriction of $\mathcal{A}$ to even functions we have therefore $\operatorname{Ker} \mathcal{A}_{e}=\{0\}$. Consequently, $\mathcal{A}_{e}: H_{e}^{2}(0, T) \rightarrow L_{e}^{2}(0, T)$ is invertible since $\mathcal{A}_{e}$ is Fredholm with index 0 . Then by Banach's isomorphism theorem it follows that $\mathcal{A}_{e}^{-1}: L_{e}^{2}(0, T) \rightarrow H_{e}^{2}(0, T)$ is continuous. This allows us to rewrite (25) in the form $u_{0}=\mathcal{F}_{\gamma, 0}\left(u_{0}, u_{1}\right)$, where $\mathcal{F}_{\gamma, 0}:\left(H_{e}^{2}(0, T)\right)^{2} \rightarrow H_{e}^{2}(0, T)$ is analytic and defined by

$$
\mathcal{F}_{\gamma, 0}\left(u_{0}, u_{1}\right)=\mathcal{A}_{e}^{-1}\left[\epsilon(\gamma)^{-1} g_{0}\left(x, \epsilon(\gamma) u_{0}\right)-U^{\prime}\left(x+\epsilon(\gamma) u_{0}\right)+\gamma^{1 / 2} u_{1}\right] .
$$

In the same way, let us reformulate (3) for $n=1$ and $n \geq 2$ using the new variables $u_{n}$. One obtains

$$
\begin{gathered}
-2 \gamma \ln \gamma \frac{\mathrm{d}^{2} u_{1}}{\mathrm{~d} t^{2}}+u_{1}=-\gamma^{-1 / 2} g\left(\gamma^{1 / 2} u_{1}\right)+\gamma\left(u_{2}-2 u_{1}\right)+\gamma^{1 / 2}\left(\tilde{y}_{0}+x+\epsilon u_{0}\right), \\
-2 \gamma \ln \gamma \frac{\mathrm{d}^{2} u_{n}}{\mathrm{~d} t^{2}}+u_{n}=-\gamma^{-1 / 2} g\left(\gamma^{1 / 2} u_{n}\right)+\gamma\left(u_{n+1}-2 u_{n}+u_{n-1}\right), \quad n \geq 2,
\end{gathered}
$$


where $g(y)=V^{\prime}(y)-y=O\left(y^{2}\right)$ as $y \rightarrow 0$. Since we choose $\gamma \in \mathcal{S}_{p}$ in Theorem 2.2, the nonresonance condition $\omega^{*}(\gamma)^{-1} \notin \mathbb{N}$ is fulfilled and the operator $\mathcal{L}_{\gamma}: H_{e}^{2}(0, T) \rightarrow$ $L_{e}^{2}(0, T), u \mapsto-2 \gamma \ln \gamma \frac{\mathrm{d}^{2} u}{\mathrm{~d} t^{2}}+u$ is invertible (with bounded inverse). Consequently we rewrite (27)-(28) in the form $u_{n}=\mathcal{F}_{\gamma, n}\left(u_{n-1}, u_{n}, u_{n+1}\right)(n \geq 1)$, where the maps $\mathcal{F}_{\gamma, n}:\left(H_{e}^{2}(0, T)\right)^{3} \rightarrow H_{e}^{2}(0, T)$ are $C^{k}$ and defined by

$$
\mathcal{F}_{\gamma, 1}\left(u_{0}, u_{1}, u_{2}\right)=\mathcal{L}_{\gamma}^{-1}\left[-\gamma^{-1 / 2} g\left(\gamma^{1 / 2} u_{1}\right)+\gamma\left(u_{2}-2 u_{1}\right)+\gamma^{1 / 2}\left(x+\epsilon(\gamma) u_{0}\right)\right]+\gamma^{1 / 2} \tilde{y}_{0},
$$

$$
\mathcal{F}_{\gamma, n}\left(u_{n-1}, u_{n}, u_{n+1}\right)=\mathcal{L}_{\gamma}^{-1}\left[-\gamma^{-1 / 2} g\left(\gamma^{1 / 2} u_{n}\right)+\gamma\left(u_{n+1}-2 u_{n}+u_{n-1}\right)\right], \quad n \geq 2 .
$$

To a sequence $u=\left(u_{n}\right)_{n \geq 0}$ in $\mathcal{E}$ we now associate the sequence $\mathcal{F}_{\gamma}(u)=\left(\mathcal{F}_{\gamma, n}(u)\right)_{n \geq 0}$ defined by (26), (29) and (30). We shall prove that $\mathcal{F}_{\gamma}$ defines a contraction on $\mathcal{E}$ for $\gamma \in \mathcal{S}_{p}$ small enough. For this purpose the first step is to estimate $\left\|\mathcal{L}_{\gamma}^{-1}\right\|_{\mathcal{L}\left(H^{2}\right)}$.

Lemma 3.1 Fix $\omega=2 \pi / T<1$. There exists a constant $C>0$ and an integer $p \geq m_{0}(\omega)$ such that for all $\gamma \in \mathcal{S}_{p}$ we have

$$
\left\|\mathcal{L}_{\gamma}^{-1}\right\|_{\mathcal{L}\left(H_{e}^{2}(0, T)\right)} \leq C \gamma^{-1 / 2}|\ln \gamma|^{r-1 / 2},
$$

where $r \in(0,1 / 2)$ gives the logarithmic decay of the function $\alpha$ defined by (18).

Proof The operator $\mathcal{L}_{\gamma}: H_{e}^{4}(0, T) \subset H_{e}^{2}(0, T) \rightarrow H_{e}^{2}(0, T)$ is unbounded, closed and self-adjoint. Its spectrum $\sigma\left(\mathcal{L}_{\gamma}\right)$ consists of simple eigenvalues $1-k^{2} \omega^{*^{2}}$ with $\omega^{*}(\gamma)=\omega \sqrt{-2 \gamma \ln (\gamma)}$, hence (see e.g. [26, p. 272])

$$
\left\|\mathcal{L}_{\gamma}^{-1}\right\|_{\mathcal{L}\left(H_{e}^{2}(0, T)\right)}=\frac{1}{\operatorname{Inf}_{k \geq 0}\left|1-k^{2} \omega^{* 2}\right|}=\frac{\mu^{2}}{f\left(\mu^{2}\right)},
$$

where we note $\mu=\omega^{*-1}>\sqrt{e / 2}$ and $f\left(\mu^{2}\right)=\operatorname{Inf}_{k \geq 0}\left|\mu^{2}-k^{2}\right|$.

If $\gamma \in \mathcal{S}_{p}$ then $\gamma \in\left[\tilde{\Gamma}_{m}, \tilde{\gamma}_{m}\right]$ for some $m \geq p$, hence $\omega^{* 2} \in\left[\Omega_{m}^{2}, \omega_{m}^{2}\right]$. This yields $\mu^{2} \in\left[m^{2}(1-\alpha(m))+(m+1)^{2} \alpha(m), m^{2} \alpha(m)+(m+1)^{2}(1-\alpha(m))\right]$ (see definition (17)), where $\alpha(m) \in\left(0, \frac{1}{2}\right)$ is defined by (18). We have thus

$$
\mu^{2} \in\left[m^{2}+(2 m+1) \alpha(m),(m+1)^{2}-(2 m+1) \alpha(m)\right],
$$

which yields $f\left(\mu^{2}\right) \geq(2 m+1) \alpha(m)$. Since $\mu-1 \leq m \leq \mu$, this ensures that $f\left(\mu^{2}\right) \geq(2 \mu-1) \alpha(\mu)$ and

$$
\left\|\mathcal{L}_{\gamma}^{-1}\right\|_{\mathcal{L}\left(H^{2}\right)} \leq \frac{\mu}{\alpha(\mu)}
$$

Since $\lim _{m \rightarrow+\infty} \tilde{\gamma}_{m}=0$ and $\lim _{m \rightarrow+\infty} \tilde{\Gamma}_{m}=0$ in the definition of $\mathcal{S}_{p}, \gamma$ can be made arbitrarily small by choosing $p$ large. Considering estimate (32) where $\mu=$ $\omega^{-1}(-2 \gamma \ln (\gamma))^{-1 / 2}$ and $\gamma \approx 0$, estimate (31) can be obtained without difficulty.

We are now ready to prove the following.

Lemma 3.2 Fix a solution $x \in H_{e}^{2}(0, T)$ of $(12)$, with period $T>2 \pi$ given by (13) and satisfying $T^{\prime}(H)>0$. There exists a constant $\rho_{0}>0$ and an integer $p$ which must be large enough, such that for all $\gamma \in \mathcal{S}_{p}$ the map $\mathcal{F}_{\gamma}$ is a contraction on $\mathcal{E}$. 
Proof As previously one can make $\gamma$ arbitrarily small by choosing $p$ large. By standard estimates (using the property $g_{0}(x, 0)=\frac{\partial g_{0}}{\partial \xi_{0}}(x, 0)=0$ and the mean value inequality), there exist constants $\rho_{x}, \kappa_{x, \rho_{0}}, \gamma_{x, \rho_{0}}>0$ such that for all $u_{0}, u_{1}, \tilde{u}_{0}, \tilde{u}_{1} \in \mathcal{E}$ and $\gamma \leq \gamma_{x, \rho_{0}}$,

$$
\begin{gathered}
\left\|\mathcal{F}_{\gamma, 0}\left(u_{0}, u_{1}\right)\right\|_{H^{2}} \leq \rho_{x}, \\
\left\|\mathcal{F}_{\gamma, 0}\left(u_{0}, u_{1}\right)-\mathcal{F}_{\gamma, 0}\left(\tilde{u}_{0}, \tilde{u}_{1}\right)\right\|_{H^{2}} \leq \kappa_{x, \rho_{0}}\left[\epsilon(\gamma)\left\|u_{0}-\tilde{u}_{0}\right\|_{H^{2}}+\gamma^{1 / 2}\left\|u_{1}-\tilde{u}_{1}\right\|_{H^{2}}\right] .
\end{gathered}
$$

Now we fix $\rho_{0}=\rho_{x}$ in the definition of $\mathcal{E}$.

Since $g(0)=g^{\prime}(0)=0$, there exist constants $\tilde{\rho}_{x}, r, \kappa_{1}, \gamma_{1}>0$ such that for all $u_{i}$, $\tilde{u_{i}} \in \mathcal{E}$, for $\gamma \in \mathcal{S}_{p}$ and $\gamma \leq \gamma_{1}$, we have

$$
\left\|\mathcal{F}_{\gamma, 1}\left(u_{0}, u_{1}, u_{2}\right)\right\|_{H^{2}} \leq \tilde{\rho}_{x} \gamma^{1 / 2}\left[\left\|\mathcal{L}_{\gamma}^{-1}\right\|_{\mathcal{L}\left(H^{2}\right)}+|\ln \gamma|\right]
$$

(use definition (7)),

$$
\begin{gathered}
\left\|\mathcal{F}_{\gamma, n}\left(u_{n-1}, u_{n}, u_{n+1}\right)\right\|_{H^{2}} \leq r \gamma^{1 / 2}\left\|\mathcal{L}_{\gamma}^{-1}\right\|_{\mathcal{L}\left(H^{2}\right)} \quad \forall n \geq 2, \\
\left\|\mathcal{F}_{\gamma, 1}\left(u_{0}, u_{1}, u_{2}\right)-\mathcal{F}_{\gamma, 1}\left(\tilde{u}_{0}, \tilde{u}_{1}, \tilde{u}_{2}\right)\right\|_{H^{2}} \\
\leq \kappa_{1}\left\|\mathcal{L}_{\gamma}^{-1}\right\|_{\mathcal{L}\left(H^{2}\right)}\left[\gamma^{1 / 2} \epsilon(\gamma)\left\|u_{0}-\tilde{u}_{0}\right\|_{H^{2}}+\gamma^{1 / 2}\left\|u_{1}-\tilde{u}_{1}\right\|_{H^{2}}+\gamma\left\|u_{2}-\tilde{u}_{2}\right\|_{H^{2}}\right]
\end{gathered}
$$

and for all $n \geq 2$

$$
\begin{aligned}
& \left\|\mathcal{F}_{\gamma, n}\left(u_{n-1}, u_{n}, u_{n+1}\right)-\mathcal{F}_{\gamma, n}\left(\tilde{u}_{n-1}, \tilde{u}_{n}, \tilde{u}_{n+1}\right)\right\|_{H^{2}} \\
& \quad \leq \kappa_{1}\left\|\mathcal{L}_{\gamma}^{-1}\right\|_{\mathcal{L}\left(H^{2}\right)}\left[\gamma\left\|u_{n-1}-\tilde{u}_{n-1}\right\|_{H^{2}}+\gamma^{1 / 2}\left\|u_{n}-\tilde{u}_{n}\right\|_{H^{2}}+\gamma\left\|u_{n+1}-\tilde{u}_{n+1}\right\|_{H^{2}}\right] .
\end{aligned}
$$

When $\gamma \rightarrow 0$ in $\mathcal{S}_{p}$, estimates (35), (36) in conjunction with Lemma 3.1 give

$$
\operatorname{Sup}_{u \in \mathcal{E}, n \geq 1}\left\|\mathcal{F}_{\gamma, n}\left(u_{n-1}, u_{n}, u_{n+1}\right)\right\|_{H^{2}}=O\left(|\ln \gamma|^{r-1 / 2}\right), \quad r \in(0,1 / 2) .
$$

With estimate (33) and $\rho_{0}=\rho_{x}$, it follows that $\mathcal{F}_{\gamma}$ maps $\mathcal{E}$ into itself for $\gamma$ small enough. From (34), (37), (38) and lemma 3.1 we find

$$
\operatorname{Sup}_{u, v \in \mathcal{E}, u \neq v} \frac{\left\|\mathcal{F}_{\gamma}(u)-\mathcal{F}_{\gamma}(v)\right\|_{\infty}}{\|u-v\|_{\infty}}=O\left(|\ln \gamma|^{r-1 / 2}\right)
$$

when $\gamma \rightarrow 0$ in $\mathcal{S}_{p}$. Consequently $\mathcal{F}_{\gamma}$ is a contraction on $\mathcal{E}$ when $\gamma \in \mathcal{S}_{p}$ and $p$ is large enough.

Now Lemma 3.2 and the contraction mapping theorem ensure that $\mathcal{F}_{\gamma}$ admits a unique fixed point $u(\gamma) \in \mathcal{E}$ for all $\gamma \in \mathcal{S}_{p}$ with $p$ large enough. Moreover, the map $(u, \gamma) \mapsto \mathcal{F}_{\gamma}(u)$ is $C^{k}$ from $\ell_{\infty}\left(\mathbb{N}_{0}, H_{e}^{2}(0, T)\right) \times \mathcal{S}_{p}$ to $\ell_{\infty}\left(\mathbb{N}_{0}, H_{e}^{2}(0, T)\right.$ ) (note that $0 \notin \mathcal{S}_{p}$ ), and $\mathcal{F}_{\gamma}$ is a contraction on $\mathcal{E}$ uniformly in $\gamma \in \mathcal{S}_{p}$ (see estimate (40)). According to the uniform contraction theorem [27, p. 107), the map $\gamma \mapsto u(\gamma)$ is then $C^{k}$ from $\mathcal{S}_{p}$ to $\ell_{\infty}\left(\mathbb{N}_{0}, H_{e}^{2}(0, T)\right)$.

The sequence $u(\gamma)$ defines a solution of Equations (25), (27), (28) by construction. Consequently, the sequence $y_{n}$ defined by (22) and

$$
\xi_{0}=-(\ln \gamma)^{-1} u_{0}(\gamma), \quad \xi_{n}=\sqrt{\gamma} u_{|n|}(\gamma)
$$


defines a time-periodic and spatially bounded solution of (3), close to the approximate breather solution (14) for $\gamma \approx 0$.

There remains to prove the spatial decay of $y_{n}$ stated in Theorem 2.2. This follows directly from the following lemma.

Lemma 3.3 There exist constants $M, K>0$ and an integer $p$ which must be large enough, such that for all $\gamma \in \mathcal{S}_{p}$, for all $n \geq 1$

$$
\left\|u_{n}(\gamma)\right\|_{H^{2}} \leq M|\ln \gamma|^{r-1 / 2}\left(K|\ln \gamma|^{r-1 / 2} \gamma^{1 / 2}\right)^{n-1}, \quad r \in\left(0, \frac{1}{2}\right) .
$$

Proof To simplify notations we omit the argument $\gamma$ of $u(\gamma)$. Since $u_{1}=\mathcal{F}_{\gamma, 1}\left(u_{0}, u_{1}, u_{2}\right)$, estimate (39) yields $\left\|u_{1}\right\|_{H^{2}}=O\left(|\ln \gamma|^{r-1 / 2}\right)$ with $r \in(0,1 / 2)$. Since $u_{n}=\mathcal{F}_{\gamma, n}\left(u_{n-1}, u_{n}, u_{n+1}\right)$, using Equation (30) and Lemma 3.1 one finds $C>0$ such that for $\gamma \in \mathcal{S}_{p}$ small enough and for all $n \geq 2$

$$
\left\|u_{n}\right\|_{H^{2}} \leq C|\ln \gamma|^{r-1 / 2}\left(\left\|u_{n}\right\|_{H^{2}}^{2}+2 \gamma^{1 / 2}\left\|u_{n}\right\|_{H^{2}}+\gamma^{1 / 2}\left\|u_{n+1}\right\|_{H^{2}}+\gamma^{1 / 2}\left\|u_{n-1}\right\|_{H^{2}}\right) .
$$

Since $\left\|u_{n}\right\|_{H^{2}} \leq 1$ we have for $\gamma$ small enough and for all $n \geq 2$

$$
\left\|u_{n}\right\|_{H^{2}} \leq 2 C|\ln \gamma|^{r-1 / 2} \gamma^{1 / 2}\left(\left\|u_{n+1}\right\|_{H^{2}}+\left\|u_{n-1}\right\|_{H^{2}}\right) .
$$

Let us note $\lambda(\gamma)=(2 C)^{-1}|\ln \gamma|^{1 / 2-r} \gamma^{-1 / 2}$ and introduce the auxiliary variable $x_{n}=\left\|u_{n}\right\|_{H^{2}}-\sigma^{n-1}\left\|u_{1}\right\|_{H^{2}}$, where $\sigma \in(0,1)$ satisfies the characteristic equation

$$
\lambda-\sigma-\sigma^{-1}=0 \text {. }
$$

Estimate (43) can be rewritten

$$
f_{n} \stackrel{\text { def }}{=} \lambda x_{n}-x_{n+1}-x_{n-1} \leq 0, \quad n \geq 2,
$$

where $\lambda>2$ for $\gamma$ small enough, $x_{1}=0$ and $\left(x_{n}\right)_{n \geq 1}$ is bounded. From the discrete maximum principle [24] it follows that $x_{n} \leq 0$ for all $n \geq 1$. This can be readily checked using the expression

$$
x_{n}=\sum_{p \geq 2} G_{n, p} f_{p},
$$

where the kernel $G_{n, p}=\left(\sigma^{-1}-\sigma\right)^{-1}\left(\sigma^{|n-p|}-\sigma^{n+p-2}\right)$ is positive. Consequently one obtains $\left\|u_{n}\right\|_{H^{2}} \leq \sigma^{n-1}\left\|u_{1}\right\|_{H^{2}}$. From (44) we get $\sigma<(\lambda-1)^{-1}<2 \lambda^{-1}$, hence estimate (41) follows.

This completes the proof of Theorem 2.2.

\section{Numerical computation of discrete breathers}

In this section, we complete the previous analytical results by numerical computations. Section 4.1 recalls a numerical continuation method which allows us to compute discrete breathers close to machine precision $[19,20]$, and explains how it must be adapted in our context where continuation from infinity has to be performed. In Section 4.2 we compute three different branches of discrete breather solutions. The first one consists of discrete breathers oscillating in the first potential 
well (these solutions have been proved to exist at small coupling using the classical anticontinuum limit [12]). The second branch corresponds to the new kind of discrete breathers described in Section 2.2, which oscillate beyond the potential barrier. The third solution branch concerns breathers oscillating on both sides of the barrier, for which no existence theorem is available yet. In addition we examine resonant cases excluded from Theorem 2.2, where discrete breathers develop an extended oscillatory tail.

Numerical computations are performed on system (1) for the on-site potential defined by

$$
V(y)= \begin{cases}A\left[e^{-\alpha y}-1\right]^{2} & \text { if } y<0, \\ a y^{2}+b y^{3}+c y^{4} & \text { if } 0 \leq y \leq 1, \\ D+E e^{-\beta y}\left(y+\frac{1}{\beta}\right) & \text { if } y>1,\end{cases}
$$

originally introduced in reference [16]. For $y<0$ this potential is identical to a Morse potential, while for large values of $y$ it decreases towards $D$ after a hump which describes the barrier for reclosing. The polynomial form for the intermediate range of $y$ provides a smooth matching between the two domains. As in reference [16], $y$ is expressed in $\AA$ and we fix $\alpha=\beta=4 \AA^{-1}, D=0.0857 \mathrm{eV}, E=4 \mathrm{eV}^{-1}$ The other parameters are calculated to ensure the continuity of the potential and of its first and second derivatives. This yields $A \approx 0.1489 \mathrm{eV}, \quad a \approx 2.3824 \mathrm{eV}^{-2}$, $b \approx-3.76263 \mathrm{eV}^{-3}$ and $c \approx 1.55751 \mathrm{eV}^{-4}$. Particle masses are set to $m=300 \mathrm{amu}$ in (1). Coupling constants $\gamma$ will be expressed in $\mathrm{eV}^{-2}$.

Remark 1 The potential defined by (45) is piecewise analytic, but only $C^{2}$ at $y=0$ and $y=1$. This lack of smoothness is not related to a physical phenomenon and corresponds to an artificial interpolation effect. The analysis of Section 3 should be adapted to include example (45), since we previously assumed $V$ at least $C^{5}$. We do not examine this problem here, since the lack of smoothness would introduce additional technicalities without physical motivation.

\subsection{Description of the numerical method}

We begin by briefly recalling classical continuation methods introduced to compute discrete breathers of prescribed period [19,20,28].

The infinite lattice (1) is replaced by a chain of $N$ oscillators with periodic boundary conditions. The problem of finding $T$-periodic solutions of (1) can be reformulated as a fixed point problem in $\mathbb{R}^{2 N}$. To a vector of initial positions and velocities $X^{0}=\left(y_{n}(0), \dot{y}_{n}(0)\right)$ we associate $\mathcal{T}\left(X^{0}\right)=X(T)$, where $X(T)$ is the value at time $T$ of the solution of the dynamical equations with initial condition $X^{0}$. A fixed point of $\mathcal{T}$ yields then a periodic orbit of (1).

Fixed points of $\mathcal{T}$ cannot be obtained by the Newton method without modifications because of the phase invariance of (1) (the fixed points of $\mathcal{T}$ are not isolated). This problem can be addressed by fixing $\dot{y}_{n}(0)=0$ for all $n$, which forces the solutions to be even in time. This approach was used in reference [28] where velocity variables were entirely discarded, yielding a restricted time- $T$ map in the subspace $\mathbb{R}^{N}$ of particle positions. However, the matrix appearing in Newton 
iterations is singular on any fixed point of this operator (a double non-semi-simple eigenvalue +1 of the Floquet matrix related to $\mathcal{T}$ yields a simple eigenvalue +1 for the restricted Floquet matrix). This leads to a rate of convergence that is only linear (whereas we would expect Newton method to be quadratic), and causes illconditioning which limits accuracy to the order of the square root of machine precision (i.e. for a computer working in double precision, $10^{-7}$ ).

To recover full convergence rate and precision, one can work instead with the restricted operator $\mathcal{R}: \mathbb{R}^{N} \rightarrow \mathbb{R}^{2 N}$ defined by $\mathcal{R}\left(Y^{0}\right)=\mathcal{T}\left(Y^{0}, 0\right)[19,20]$. Considering $\mathcal{F}(Y)=\mathcal{R}(Y)-(Y, 0)$, the above fixed-point problem is equivalent to minimizing $\|\mathcal{F}(Y)\|_{2}$ (since we know that this quantity vanishes on any solution). This problem can be solved iteratively using the standard Gauss-Newton algorithm. Given $Y_{k} \in \mathbb{R}^{N}$, we choose $Y_{k+1} \in \mathbb{R}^{N}$ so as to minimize the quantity $\| \mathcal{F}\left(Y_{k}\right)+$ $\partial \mathcal{F}\left(Y_{k}\right)\left(Y_{k+1}-Y_{k}\right) \|_{2}$ (classical linear least squares problem). Given a good initial guess $Y_{0}$, one iterates this procedure until convergence is obtained. This method does not have the limitation in precision of the previous method, and can be proven to have quadratic convergence if the starting point $Y_{0}$ is close enough to a solution. In addition, it is useful to modify the Gauss-Newton scheme using Broyden method [29], as the evaluation of the Jacobian $\partial \mathcal{R}$ (whose computation time is roughly $N$ times that of $\mathcal{R}$ ) is the bottleneck of the algorithm.

However, this method needs to be modified in the present case where solution branches are continued from infinity, on the flat part of the on-site potential $V$. As observed in [17] (see also Figure 5), the period of breathers oscillating beyond the barrier or on both sides varies in an extremely narrow band. This leads to illconditioning when $T$ is prescribed and forces to guess a suitable $T$ beforehand. To compute such solutions it is therefore more convenient to include the period $T$ as a

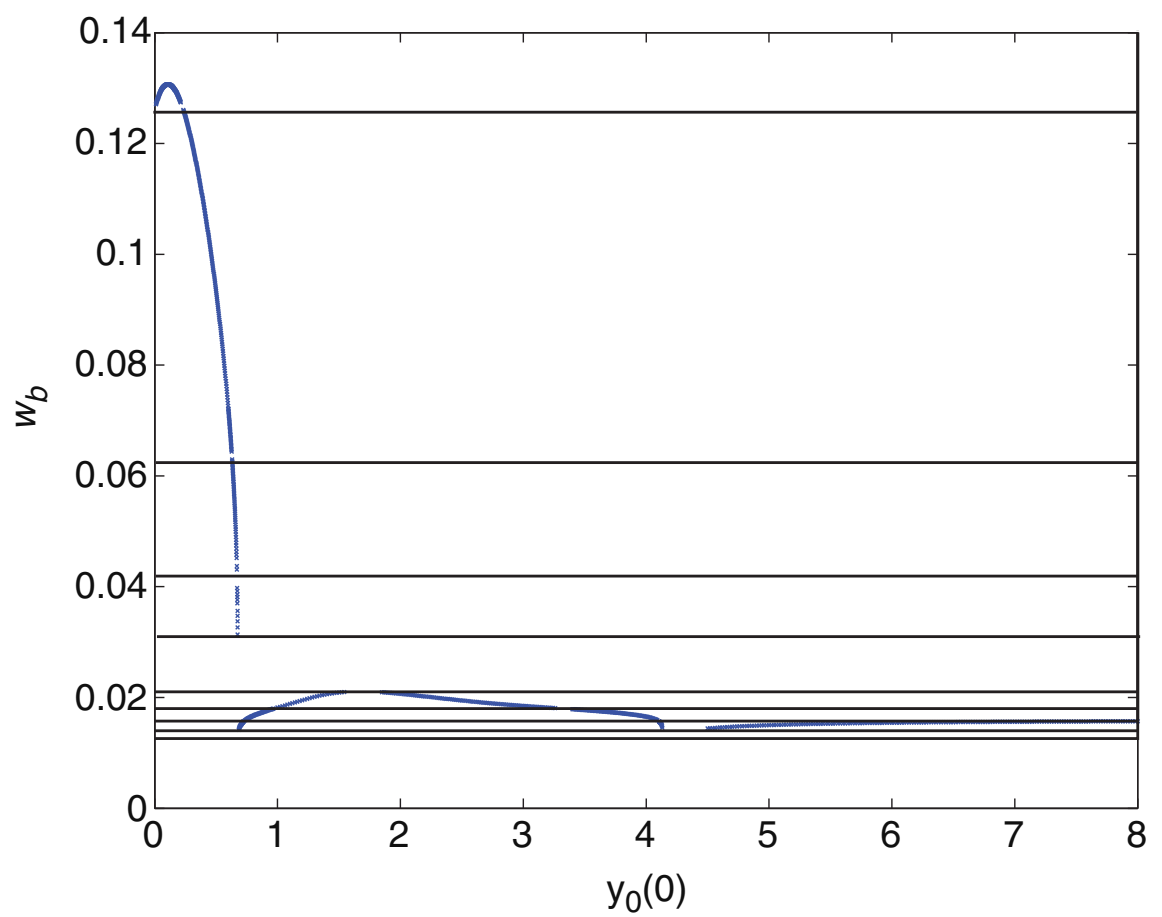

Figure 5. Frequency $\omega_{b}$ of breather solutions as a function of the amplitude $y_{0}(0)$ at the central site. Resonances with phonons, represented by black bands, prevent the existence of breathers (only the first 10 resonance bands have been represented for clarity). The coupling constant $\gamma$ is fixed to 0.01 . 
variable in the Newton method, and prescribe the amplitude $y_{0}(0)$ of the breather instead. Then we set $T=2 \pi / \Omega$ to initiate the Newton method, where

$$
\Omega=\left[\frac{V_{\gamma}^{\prime \prime}\left(\tilde{y}_{0}\right)}{m}\right]^{1 / 2}=\left[-2 \frac{\gamma}{m} \ln \left(\frac{2 \gamma}{\beta E}\right)\right]^{1 / 2}
$$

approximates for $\gamma \approx 0$ a localized mode frequency in the limit of small amplitude oscillations around the localized equilibrium $y_{n}^{\mathrm{eq}}$ [17]. Solution branches are constructed by path-following, i.e. we slowly vary amplitude and use the previous breather as a new initial condition.

\subsection{Numerical continuation of discrete breathers}

In this section, we use the numerical method described in Section 4.1 to compute branches of discrete breathers solutions. Our computations are performed on a lattice with 40 sites and periodic boundary conditions. Iterations are stopped when $\left\|\mathcal{F}\left(Y_{k}\right)\right\|_{\infty}$ and $\left\|Y_{k+1}-Y_{k}\right\|_{\infty}$ drop below an error tolerance of $10^{-8}$ (Section 4.1).

Figure 5 represents the frequency of discrete breathers as a function of their amplitude $y_{0}(0)$, for $\gamma=0.01$. Three branches of solutions can be identified. The first one $\left(y_{0}<0.7\right)$ corresponds to breathers oscillating below the potential barrier. Such solutions are well known [28], but we can note here an unusual nonmonotonic dependence of frequency versus amplitude in the neighbourhood of $y_{0}=0$, due to the fact that $V$ is not $C^{3}$ at the origin. The second branch $\left(0.7<y_{0}<4.5\right)$ corresponds to breathers oscillating beyond the barrier, described in Section 2.2 and in Theorem 2.2 for smooth potentials. The last solution branch $\left(y_{0}>4.5\right)$ yields breathers oscillating on both sides of the barrier, i.e. $y_{0}(t)$ ranges between the maximal value $y_{0}(0)>4.5$ and a negative minimal value. The existence of these solutions is still an open mathematical problem.

For $y_{0} \approx 0.7$ and $y_{0} \approx 4.5$ we observe sudden rises in period. We hypothesize a vanishing of the frequency at two particular values, in the neighbourhood of which discrete breathers spend a long time near an unstable localized equilibrium, forming nearly homoclinic orbits. Note that frequency is computed for prescribed amplitudes in our numerical scheme (Section 4.1). This method is well suited to compute the new solution branches originating from the potential barrier effect (since frequency variations are very small along them), but the scheme is not efficient in the regions where frequency varies rapidly with amplitude and many resonances occur (see below). The missing parts of the curves in Figure 5 correspond to such parameter regimes.

To intuitively understand these results we consider the limiting case when only the central site $n=0$ oscillates, the other sites being kept to 0 . In this case, breathers oscillate in the potential $V_{\gamma}$ represented in Figure 3. We obtain a double-well potential, and the branches of breather solutions of Figure 5 correspond to oscillations in a given well or in the two wells.

Now let us focus on the discrete breathers oscillating beyond the potential barrier, which we believe to play a key role in the dynamics of DNA breathing [17]. Figure 6 provides the temporal profile of this type of solution in a strongly anharmonic regime. This profile is compared with the slightly improved version of 


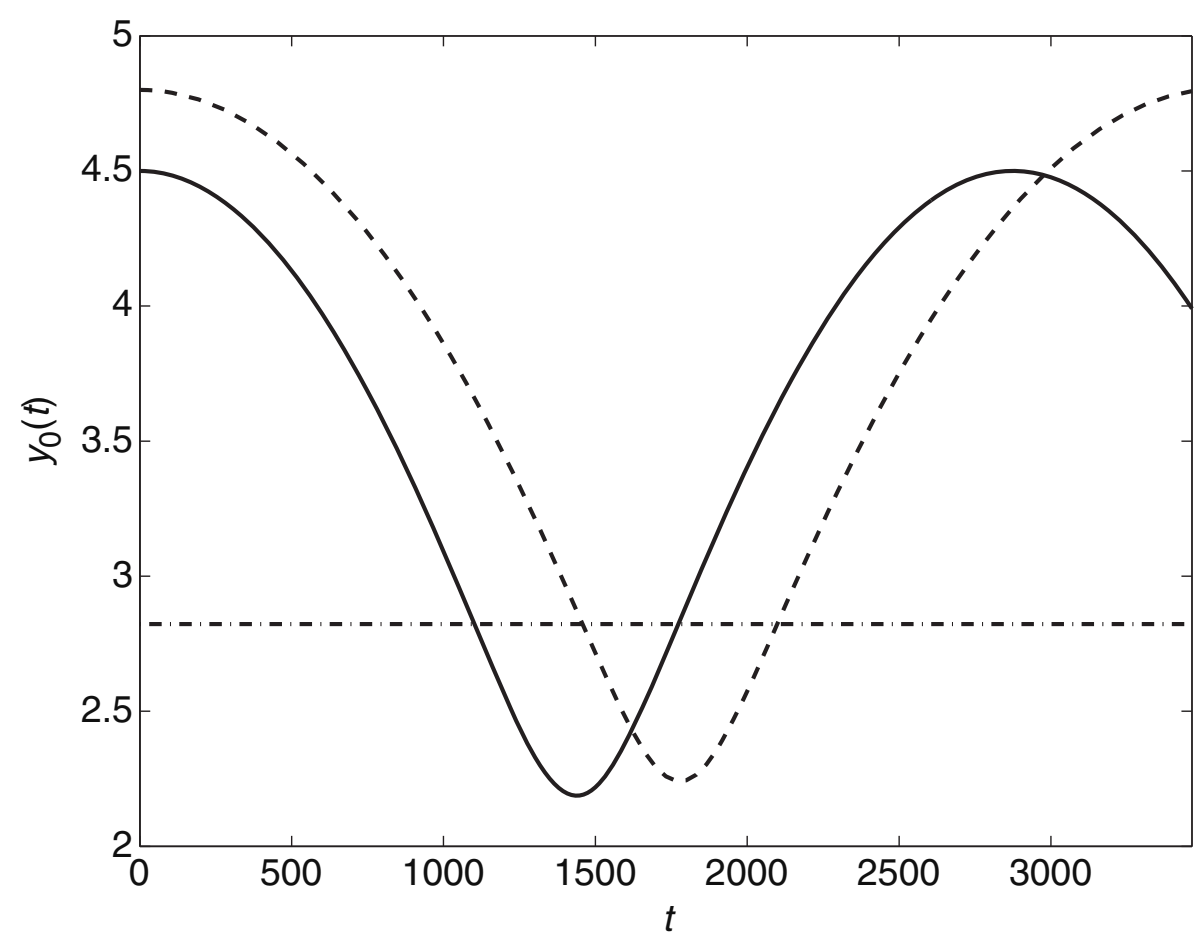

Figure 6. Temporal profile $y_{0}(t)$ of a discrete breather oscillating beyond the potential barrier, obtained for $\gamma=10^{-4}$. The solid curve corresponds to a numerically computed breather, and the dashed curve to an approximate solution given by (47). The dash-dot line indicates the localized equilibrium $y_{0}^{\mathrm{eq}}$.

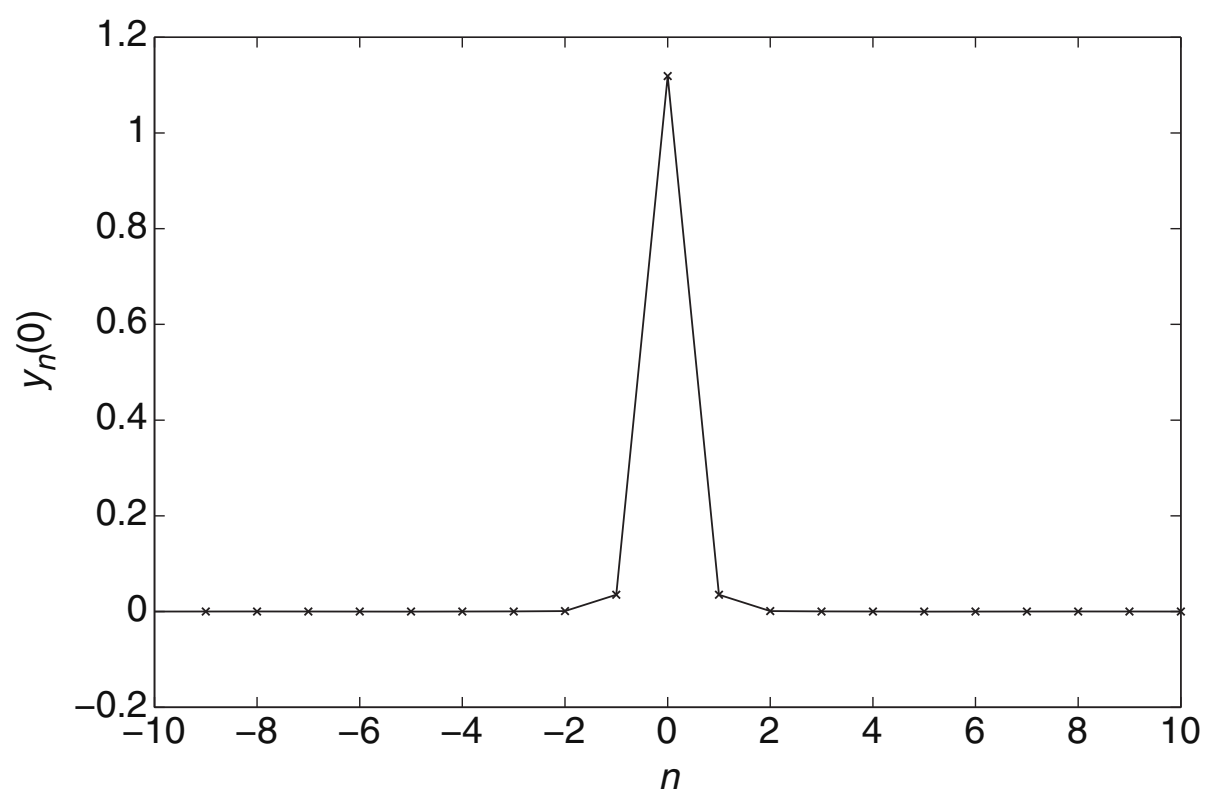

Figure 7. Spatial profile $y_{n}(0)$ of a discrete breather oscillating beyond the potential barrier, obtained for $\gamma=0.14$.

approximation (14)

$$
y_{n}^{*}(t)=\delta_{n, 0}\left[-\frac{1}{\beta} \ln \left(\frac{2 \gamma}{\beta E}\right)+x(\Omega t)\right],
$$

where $\Omega$ is defined by (46) and $x$ is a periodic solution of (12) (note that the frequencies of the approximate solutions (47) and (14) coincide at leading order in $\gamma$, but their frequency mismatch is $O\left(|\ln (\gamma)|^{-1}\right)$ and decays slowly as $\left.\gamma \rightarrow 0\right)$. Approximation (47) reproduces fairly well the parabolic profile of breather 

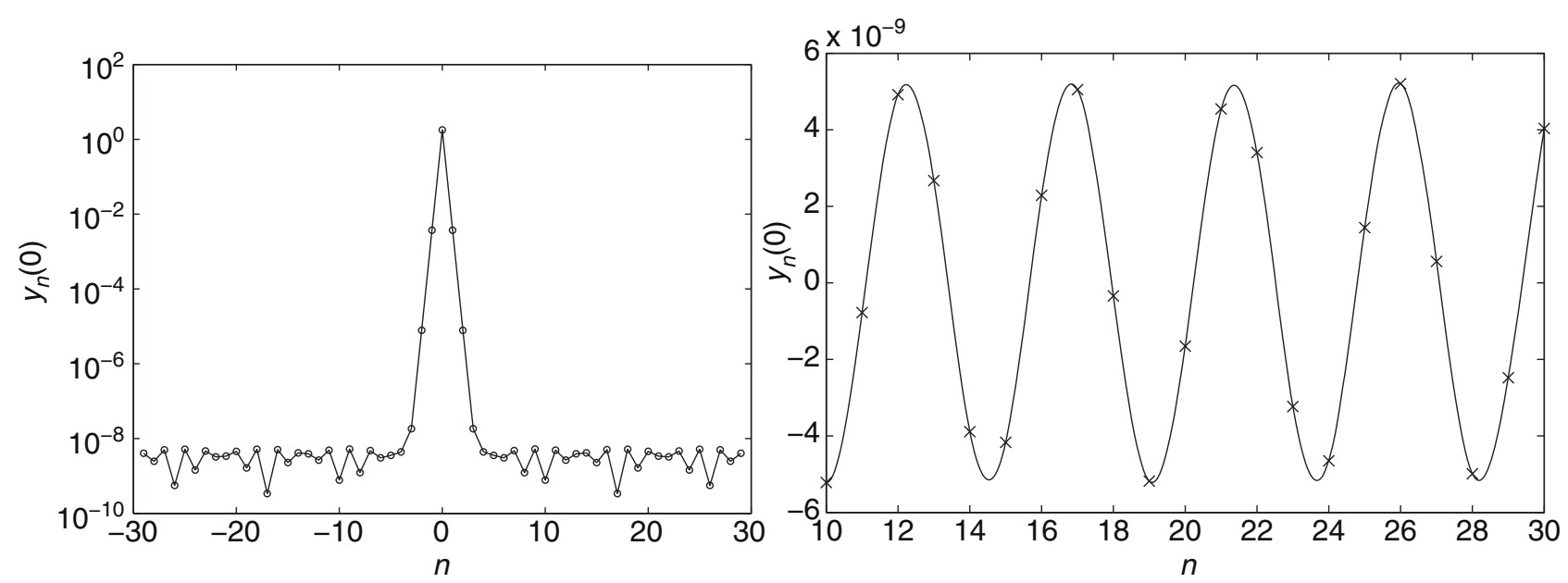

Figure 8. Spatial profile of a resonant breather solution, superposed on a small oscillatory tail. The sixth harmonic of the breather belongs to the phonon band. The coupling constant is $\gamma=0.01$. The left plot gives the solution profile in semilogarithmic scale. The right plot shows the details of the oscillatory tail (normal scale). A spline interpolant (continuous line) reveals the periodicity of the spatial oscillations, which involve only one normal mode.

oscillations (this profile originates from the asymptotically linear behaviour of the renormalized potential $\Phi$ at $+\infty$ ), but it only provides a crude approximation of breather solutions with a logarithmic convergence as $\gamma \rightarrow 0$. Figure 7 provides the spatial profile of a discrete breather of the same type, showing its strong spatial localization.

In Figure 5, each branch of discrete breathers is interrupted when it enters the phonon band or one of its submultiples (thin black bands in Figure 5), but it can be continued again when leaving the resonance band. In resonant cases (i.e. when a multiple of the breather frequency belongs to the phonon band), the breather generally loses its spatial localization and is superposed on a small nondecaying oscillatory tail as $n \rightarrow \pm \infty$. Figure 8 represents such oscillations for a lattice of 60 sites and $\gamma=0.01$. Decreasing our error tolerance to $10^{-13}$, we observe that the oscillatory tail is generally very small, typically of the order of $10^{-8}$.

\section{Acknowledgements}

C. Ferreira acknowledges support from the CAPES (Coordenação de Aperfeiçoamento de Pessoal de Nivel Superior-Brasil, www.capes.gov.br). This work was supported by the CNRS program PEPS PTI (Physique Théorique et ses Interfaces).

\section{References}

[1] M. Peyrard and A.R. Bishop, Statistical mechanics of a nonlinear model for DNA denaturation, Phys. Rev. Lett. 62 (1989), p. 2755.

[2] M. Peyrard, Nonlinear dynamics and statistical physics of DNA, Nonlinearity 17 (2004), pp. R1-R40.

[3] T. Dauxois, M. Peyrard, and A.R. Bishop, Entropy-driven DNA denaturation, Phys. Rev. E 47(1) (1993), pp. R44-R47.

[4] S. Flach and A. Gorbach, Discrete Breathers: Advances in Theory and Applications, Phys. Rep. 467 (2008), pp. 1-116. 
[5] I. Daumont, T. Dauxois, and M. Peyrard, Modulational instability: First step towards energy localization in nonlinear lattices, Nonlinearity 10 (1997), pp. 617-630.

[6] M.V. Ivanchenko, O.I. Kanakov, V.D. Shalfeev, and S. Flach, Discrete breathers in transient processes and thermal equilibrium, Phys. D 198 (2004), pp. 120-135.

[7] G. Arioli and A. Szulkin, Periodic motions of an infinite lattice of particles: The strongly indefinite case, Ann. Sci. Math. Québec 22 (1998), pp. 97-119.

[8] S. Aubry, Breathers in nonlinear lattices: Existence, linear stability and quantization, Phys. D 103 (1997), pp. 201-250.

[9] S. Aubry, G. Kopidakis, and V. Kadelburg, Variational proof for hard discrete breathers in some classes of Hamiltonian dynamical systems, Discrete Contin. Dyn. Syst. B 1 (2001), pp. 271-298.

[10] G. James, Centre manifold reduction for quasilinear discrete systems, J. Nonlinear Sci. 13 (2003), pp. 27-63.

[11] G. James, B. Sánchez-Rey, and J. Cuevas, Breathers in inhomogeneous lattices: An analysis via center manifold reduction, Rev. Math. Phys. 21 (2009), pp. 1-59.

[12] R.S. MacKay and S. Aubry, Proof of existence of breathers for time-reversible or Hamiltonian networks of weakly coupled oscillators, Nonlinearity 7 (1994), pp. 1623-1643.

[13] A. Pankov, Travelling waves and Periodic Oscillations in Fermi-Pasta-Ulam Lattices, Imperial College Press, London, 2005.

[14] J.-A. Sepulchre and R.S. MacKay, Localized oscillations in conservative or dissipative networks of weakly coupled autonomous oscillators, Nonlinearity 10 (1997), pp. 679-713.

[15] A. Zevin, Localization of periodic oscillations in discrete non-linear systems, J. Sound Vibration 193 (1996), p. 847.

[16] M. Peyrard, S. Cuesta-López, and G. James, Modelling DNA at the mesoscale: A challenge for nonlinear science?, Nonlinearity 21 (2008), pp. T91-T100.

[17] M. Peyrard, S. Cuesta-López, and G. James, Nonlinear analysis of the dynamics of DNA breathing, J. Biol. Phys. 35 (2009), pp. 73-89.

[18] J. Fura and S. Rybicki, Periodic solutions of second order Hamiltonian systems bifurcating from infinity, Ann. Inst. Henri Poincaré (C) Anal. Non Linéaire 24(3) (2007), pp. 471-490.

[19] D. Chen, S. Aubry, and G.P. Tsironis, Breather Mobility in Discrete $\phi^{4}$ Nonlinear Lattices, Phys. Rev. Lett. 77 (1996), pp. 4776-4779.

[20] T. Cretegny and S. Aubry, Spatially inhomogeneous time-periodic propagating waves in anharmonic systems, Phys. Rev. B 55 (1997), pp. R11929-R11932.

[21] A.M. Morgante, M. Johansson, S. Aubry, and G. Kopidakis, Breather-phonon resonances in finite-size lattices: 'Phantom breathers'?, J. Phys. A: Math. Gen. 35 (2002), pp. 1-23.

[22] S. Aubry and G. Abramovici, Chaotic trajectories in the standard map, The concept of antiintegrability, Phys. D 43 (1990), pp. 199-219.

[23] S. Aubry, Anti-integrability in dynamical and variational problems, Physica D 86 (1995), pp. 284-296.

[24] P.G. Ciarlet, Discrete maximum principle for finite-difference operators, Aequationes Math. 4 (1970), pp. 338-352.

[25] V.I. Arnold, Ordinary Differential Equations, Springer, Berlin, 1992.

[26] T. Kato, Perturbation Theory for Linear Operators, Springer Verlag, Berlin, 1980.

[27] C. Chicone, Ordinary Differential Equations with Applications, Texts in Applied Mathematics, Vol. 34, Springer, New York, 1999.

[28] J.L. Marín and S. Aubry, Breathers in nonlinear lattices: Numerical calculation from the anticontinuous limit, Nonlinearity 9 (1996), pp. 1501-1528.

[29] J.E. Dennis Jr and R.B. Schnabel, Numerical methods for unconstrained optimization and nonlinear equations, Classics in Applied Mathematics, Vol. 16, SIAM, Philadelphia, 1996. 